\title{
Aportaciones al análisis comparativo entre modelos de desarrollo rural en Europa
}

Contributions to the comparative analysis of models of rural development in Europe

\author{
Alberto Pérez Chueca \\ Grupo de Investigación "Territorio, Cultura y Desarrollo" (TECUDE). Universidad de Sevilla. España \\ achueca@us.es
}

\section{Encarnación Aguilar}

Antropóloga, Departamento de Antropología Social, Universidad de Sevilla (España)

eaguilar@us.es

\section{NUEVAS RURALIDADES}

MONOGRÁFICO COORDINADO POR SHARON R. ROSEMAN, SANTIAGO PRADO Y XERARDO PEREIRO

\begin{abstract}
RESUMEN
El objetivo de este artículo es presentar el marco teórico-metodológico propuesto para la realización de un análisis comparativo de casos de desarrollo rural a nivel europeo. Dada la naturaleza y los objetivos de la investigación este marco teórico metodológico se centra en tres dimensiones: organizativa-institucional, territorial e identitaria; que corresponden a los principales procesos de transformación que han ido teniendo lugar durante los últimos años como consecuencia de la implementación de las políticas de desarrollo rural europeas. Utilizando un enfoque de redes sociales como analizamos cada una de estas tres dimensiones y profundizamos en su reflexión haciendo uso de los conceptos de capital social y capital institucional. Pretendemos proponer un marco teórico metodológico adecuado para abordar la gran diversidad de modelos de desarrollo rural existentes en la actualidad en el contexto de la Unión Europea, aportando con ello una valiosa herramienta para entender algunas de las claves de la nueva ruralidad europea.
\end{abstract}

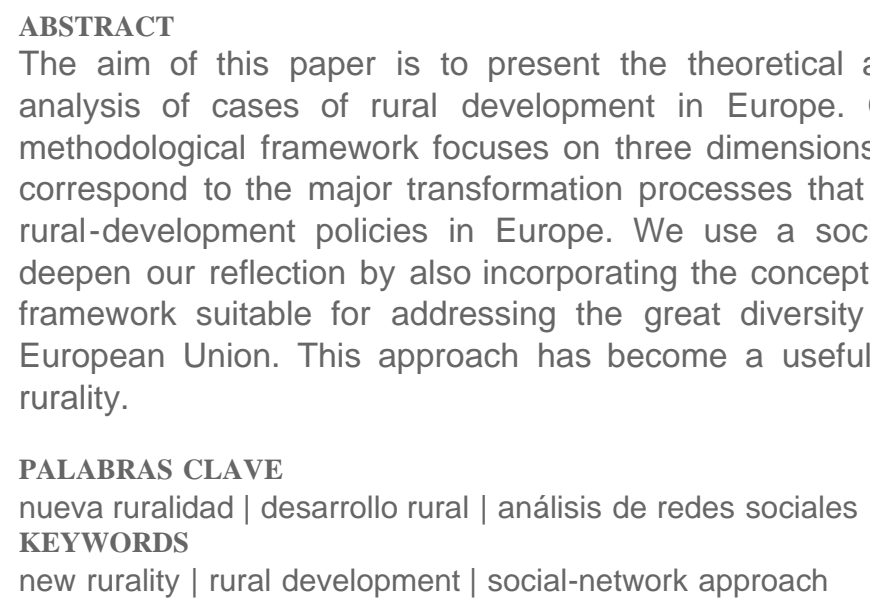
rurality. analysis of cases of rural development in Europe. Given the nature and objectives of this research, the theoretical and methodological framework focuses on three dimensions: organizational - institutional, territorial, and identity. These dimensions correspond to the major transformation processes that have been taking place in recent years following the implementation of rural-development policies in Europe. We use a social-network approach to discuss each of these three dimensions and deepen our reflection by also incorporating the concepts of social capital and institutional capital. We propose a methodological framework suitable for addressing the great diversity of rural-development models currently existing in the context of the European Union. This approach has become a useful tool for understanding some of the keys aspects of the new European

\section{La nueva ruralidad: transformaciones y posicionamientos teóricos en la caracterización de los espacios rurales contemporáneos (1)}

Las importantes transformaciones acaecidas durante las últimas décadas en nuestra sociedad a nivel global, se han reflejado lógicamente en la profunda reestructuración de la sociedad rural. Una nueva realidad ha requerido, necesariamente, la revisión de los paradigmas teóricos que han venido explicando las sociedades rurales. Podemos afirmar por ello que en las dos útlimas décadas vivimos una doble transformación de las sociedades rurales: la del propio objeto de estudio y la de nuestra forma de mirarlo. 
Dejando atrás la definición de lo rural como lo agrario y "no urbano", se empieza a forjar una nueva ruralidad, articulada e interrelacionada con los procesos de cambio global a nivel económico, social y cultural. La ruralidad se convierte hoy, como todo en la era del post - consumo, en producto del mercado y en objeto, por tanto, de múltiples intereses. Lo rural adquiere nuevas funciones derivadas de las nuevas demandas de la sociedad de consumo postmoderna y globalizada.

A partir de la década de los 80 , nuevas corrientes teóricas de la sociología rural han tratado de analizar estos profundos cambios que afectan a las zonas rurales con objeto de encauzar las políticas de desarrollo que se implementan en estos espacios.

La corriente de la Reestructuración Rural (2) parte de la idea de que los nuevos cambios acaecidos en los espacios rurales producen una reestructuración del espacio, fundamentalmente de las actividades económicas adquiriendo relevancia nuevos sectores (como el turismo, la agroalimentación, la repostería, la artesanía, la protección ambiental) en detrimento de actividades desarrolladas tradicionalmente (básicamente, la agricultura y la ganadería). Por ello aplicarán un enfoque multidisciplinar que permite analizar las diferentes dimensiones (social, política, económica, geográfica) en las que se manifiesta esta reestructuración: contraurbanización, reestructuración agraria, diversificación económica, movilidad laboral (Lowe et al. 1993) (3).

El énfasis de esta perspectiva estará en el estudio de las características que adopta una ruralidad crecientemente segmentada como resultado de su inserción en dinámicas de alcance global (Oliva 1995), así como en el análisis de las formas que adquieren las expresiones locales de la globalización (Aguilar y Bueno 2003) en los espacios rurales, de forma que aporta una visión de lo rural compleja y globalizadora (González 2002).

La crítica más común que se ha realizado a esta corriente ha sido su atención en las actividades económicas, olvidando la importancia de lo sociocultural y lo sociopolítico (Moscoso; 2007), sin integrar, además, dimensiones como la clase, la etnia, el género o el análisis de los procesos de cambio global (Camarero y González 2005).

Una segunda corriente, el Constructivismo aparece en los primeros años 90 (4) proponiendo un análisis más complejo sobre las transformaciones del mundo rural en el que se articulan "elementos económicos, sociales y políticos que pueden estar presentes a escala local, regional, nacional e internacional" (Marsden et al. 1993). La idea fundamental que transmite esta nueva corriente, y que ya había sido enunciada por Mormont (1990), es destacar que el concepto de lo rural forma parte de las representaciones construidas por las diferentes sociedades en cada momento histórico.

Desde esta corriente se enfatiza la interdependencia entre factores económicos, políticos y sociales, a nivel global y local, en la configuración de los espacios rurales; que son entendidos como construcciones sociales (5). Una de las aportaciones originales de esta corriente es la preeminencia que otorgan a los factores culturales en el proceso. De este modo, proponen como lo cultural, que se consume en forma de signos y significados, modela y construye nuevas realidades; realidades que a su vez determinan diferentes formas de organización del espacio (Lash y Urry 1998).

A través de esta argumentación teórica se consolida esta corriente que concibe lo rural como una construcción social, como el "resultado de la acción social" (González y Camarero 1999: 57), como una representación social del espacio (Halfacree 1993), y como un discurso (Marsden et al. 1993). De este modo, esta perspectiva potencia el análisis de las redes de actores involucrados en la construcción de estas representaciones, puesto que: "La interpretación que ofrece el enfoque constructivista hace aflorar el carácter intersubjetivo y sociopolítico de la categorización de determinadas partes del espacio como rurales y las pugnas reales y simbólicas que se producen en la definición global y local de éstas" (González 2002: 86).

La profunda transformación que la imagen de la ruralidad ha sufrido en los últimos años representa un fenómeno intrínsicamente ligado a la función que desarrolla lo rural en el imaginario cultural e ideológico 
de la postmodernidad en el que se produce (6). De este modo, las nuevas funciones asociadas a lo rural como objeto de consumo guiarán las transformaciones sufridas en estos espacios. Estos nuevos procesos de cambio en los que se inscribe la ruralidad en la actualidad han sido denominados como "nueva ruralidad" (Bradshaw y Blakely 1979), "post-rural" (Murdoch y Pratt 1993) o "ruralidad postproductivista" (Halfacree 1997).

Nuevas aproximaciones que tratan de explicar los elementos esenciales de esta nueva ruralidad, que al edificarse sobre la estructura del paradigma del desarrollo aportan novedosos elementos clave a la configuración de modelos de desarrollo rural a escala local. Uno de ellos es el acento en la dimensión territorial, en contraste a la dimensión sectorial agraria dominante en la definición de los espacios rurales a lo largo de la historia. El elemento agrario asociado a la ruralidad, que continúa siendo importante en el análisis de los espacios rurales, sufre una importante transformación al reconocerse las distintas funciones y servicios prestados por la agricultura a la sociedad más allá de los aspectos productivos (multifuncionalidad)(7) y al tomar conciencia de la complementariedad de la actividad agrícola con otras actividades para la generación de ingresos en la zona rural (pluriactividad y la diversificación).

Otro de los elementos sobre los que se asienta esta nueva ruralidad será el reconocimiento de la integración de las zonas rurales en los mercados y en el proceso de globalización, señalando cómo las transformaciones sociales, económicas y culturales acaecidas en los espacios rurales se articulan dentro de este proceso, constituyendo el reflejo localizado y micro de tendencias globales. La integración en el mercado de las zonas rurales hace subrayar el potencial económico que ofrecen los activos ligados al territorio, tanto de tipo geográfico, como histórico, cultural, paisajístico, ecológico o de calidad de vida. En este sentido se refuerza la noción de competitividad territorial (8) frente a la sectorial como objetivo de las políticas de desarrollo de las zonas rurales.

El reconocimiento de los múltiples vínculos entre el desarrollo urbano y rural constituye otro elemento de caracterización de la nueva ruralidad que destaca la superación de la dicotomía rural/urbano. De este modo, la articulación entre las ciudades y sus entornos circundantes, o entre agrupaciones de municipios rurales de diferente tamaño refuerzan la visión territorial del desarrollo y su cambio de escala, la cual pasa de estar basada en el municipio a estarlo sobre un conjunto de municipios organizados en torno a estrategias de cooperación territorial.

La mejora de los transportes, las comunicaciones y la movilidad hace que se tenga una mayor conciencia de la función residencial de las zonas rurales, en oposición a una percepción más relacionada con una función productiva o recreativa de las mismas. Como consecuencia de ello se presta una mayor atención a la provisión de servicios públicos básicos en las zonas rurales como reflejo de la calidad de vida al que pueden aspirar sus habitantes presentes y futuros y como un indicador de cohesión entre los distintos territorios rurales y urbanos.

Un último factor sobre el que se define la nueva ruralidad hace referencia a la participación de diversos actores involucrados desde distintos niveles en el diseño y la aplicación de las políticas y programas de desarrollo rural. Participación que queda patente en la creación de mecanismos y dinámicas de concertación de intereses entre los actores locales y en la implicación de los distintos niveles administrativos supraestatales, estatales, regionales y locales en el desarrollo de políticas y acciones de desarrollo rural. Esta nueva dinámica de implementación de las iniciativas de desarrollo queda marcada por un enfoque descendente en el que el desarrollo rural pasa a formar parte integrante de las agendas políticas de los distintos niveles administrativos y paralelamente cobran protagonismo los enfoques ascendentes y participativos en los que los actores locales toman una mayor conciencia de su papel en los procesos de toma de decisiones sobre el desarrollo de sus territorios.

\section{Un enfoque comparativo para el análisis de procesos de desarrollo rural en la Unión Europea}

Aunque los objetivos del análisis comparativo sean análogos a los del método científico en general, este tipo de análisis requiere un método propio que se diferencia en su orientación y procedimientos de los de 
En términos generales el método comparativo consiste en la utilización sistemática de observaciones extraídas de dos o más entidades sociales, para examinar sus semejanzas y diferencias e indagar sobre las causas de éstas (Elder 1976, Lijphart 1971), siendo por ello un método de análisis idóneo para la investigación en el marco del desarrollo rural europeo, donde se implementan ciertas tendencias generales de promoción del desarrollo en realidades locales diferentes.

Desde esta perspectiva adquieren especial relevancia los elementos contextuales homogéneos o heterogéneos que influyen en los casos seleccionadas para nuestra investigación(9). Por este motivo, el análisis del contexto en el que cada caso está inmerso resulta esencial puesto que, no sólo evidencia la comparabilidad entre ellos, sino que también perfila las áreas, temas (subconjuntos) sobre las que establecer esta comparación.

La Globalización es un referente contextual ineludible en la realización de cualquier investigación social actual. Su característica fundamental consiste en la integración de las diversas sociedades en un único mercado capitalista mundial, y lo que es más importante, hace funcionar sus procesos de desarrollo bajo una misma lógica capitalista y de consumo. Esta lógica impregna tanto los ámbitos económicos como los sociales, culturales y territoriales, de modo que ciertos elementos (identidad, especificidad territorial, estilos de vida) se definen como activos potenciales para el desarrollo.

Como consecuencia de la globalización y de la expansión de la lógica de mercado, nuevas demandas de la sociedad de consumo definen nuevas funciones asociadas a los espacios rurales en la actualidad. Esta nueva ruralidad constituye otro de los elementos de contexto esenciales para la investigación de los procesos de desarrollo rural puesto que los variados factores que la describen generan importantes repercusiones e impactos a nivel social, cultural, económico y territorial en las zonas rurales europeas. Tal y como proponen no pocos autores (Ploeg et al. 2000)(10), es sólo mediante el análisis centrado en el desarrollo, entendido como proceso de cambio social, como podemos dilucidar con rigor las implicaciones de este nuevo paradigma y las transformaciones por él generadas.

El desarrollo rural ha pasado a constituir una parte cada vez más importante de las agendas políticas en Europa. Por ello, otro elemento contextual ineludible para nuestra investigación será el marco político y normativo sobre el desarrollo rural de la Unión Europea. A este respecto destacamos la gran importancia de la implementación del enfoque LEADER en las políticas comunitarias encargadas de regular el desarrollo rural (11) en la Unión Europea.

Los principios que rigen este enfoque representan las nuevas orientaciones generales sobre el desarrollo rural y exponen las claves del modelo "europeo" de promoción del desarrollo rural que se centran en el enfoque territorial de los procesos de desarrollo, los partenariados locales público - privados como organizaciones dinamizadoras de estos procesos, el carácter ascendente que potencia la participación de los actores locales, el carácter integrado y multisectorial desde el que se incluyen todos los sectores económicos en el proceso, el carácter innovativo, la creación de redes y la descentralización financiera (Champentier 1999).

Estos principios del enfoque LEADER se articulan en los procesos de desarrollo local con factores clave como el entorno institucional (12), los agentes locales y el contexto rural, de modo que modifica sustancialmente los enfoques tradicionales en la aplicación de programas innovadores de desarrollo. Como expone Farell (2003), el LEADER se caracteriza, en síntesis, por ser un método innovador para el desarrollo rural que está basado en dos ideas principales. La proximidad en relación a los territorios (enfoque territorial), a los ciudadanos (enfoque desde abajo) y a las actividades (gestión descentralizada de las decisiones y las financiaciones); y la creación de enlaces entre los territorios (la organización en redes), entre los ciudadanos y las organizaciones (la asociación horizontal y público-privado y la creación del Grupo de Acción Local), y entre las actividades (el método integrado o multi-sectorial).

Además de la atención a estos elementos contextuales, el rasgo más importante del método comparativo es el uso de conceptos comparativos que sean capaces de viajar (Sartori 1970), es decir, que sean 
aplicables a más de un país o cultura. Desde nuestra perspectiva de investigación el enfoque LEADER y su método específico de actuación generan profundas transformaciones sociales, culturales y territoriales en los procesos de desarrollo rural a escala comunitaria. Todo este conjunto de transformaciones son planteadas en torno a tres dimensiones: organizativa-institucional, territorial e identitaria que constituyen la base para establecer comparaciones sistemáticas entre una gran diversidad de casos y modelos de desarrollo rural a escala local.

\subsection{La dimensión organizativa e institucional del desarrollo rural}

Una de las principales transformaciones que tienen lugar en la actualidad en los espacios rurales europeos se basa en el incremento de los actores de todo tipo que participan en los procesos de desarrollo y en la formulación de nuevas dinámicas de cooperación, coordinación y concertación entre esta renovada red de actores. Hacemos con ello referencia, siguiendo los presupuestos teóricos de Douglas North (1984 1990) a la esfera de las instituciones, entendidas como un conjunto de "reglas del juego" que regularían la interacción y los procedimientos entre los individuos (actores u organizaciones) para la consecución de un objetivo común; y las organizaciones, que serán entendidas como los "jugadores" que interaccionan en base a estas reglas. Ambas elementos (organizaciones e instituciones) conformarían sistemas institucionales (Prats 2004) que dotan de eficiencia y equidad al grupo social implicado en el proceso.

Tanto por su enfoque territorial como por su carácter ascendente y, sobretodo, por la importancia dada a la creación de partenariados locales público-privados, el LEADER ha sido origen de sustanciales modificaciones en los procesos de planificación participativa y con escala territorial del desarrollo rural. Los Grupos de Acción Local (GAL) adquieren un papel preponderante en estos procesos al constituir la organización básica de este enfoque sobre las que recae la responsabilidad de elaborar el plan de acción local de forma participativa en el que se definen las estrategias de desarrollo prioritarias para el territorio en un periodo de tiempo establecido. El Consejo Territorial de Desarrollo, como organismo rector del GAL, cumple la función de evaluar y conceder la financiación a las iniciativas locales resaltando cierta autonomía financiera y de decisión a nivel subregional aportada por este enfoque (13).

El método trata de romper la demarcación entre responsables de las intervenciones y potenciales beneficiarios configurando con ello nuevas redes de cooperación y coordinación y potenciando la ampliación y diversificación de los actores implicados en los procesos de desarrollo.

Tal y como se ha venido resaltando en las evaluaciones de la iniciativa comunitaria LEADER, una de las consecuencias de su aplicación ha sido el incremento del número de actores sociales implicados en estos contextos (14) y el refuerzo del protagonismo de estos actores locales en la toma de decisiones. De este modo, se pone de manifiesto la ampliación de la red de actores implicados en estos procesos y su pluralidad puesto que existe una apertura a actores de todos los sectores económicos y sociales del territorio, así como a los actores institucionales (15) de distintos niveles.

Con la articulación de nuevas relaciones e interacciones entre actores diversos en espacios de concertación a nivel territorial, se incide en la puesta en marcha de nuevos roles, funciones y posibilidades de interacción entre dos grandes grupos de actores: los actores sociales y los actores institucionales que son movilizados en los procesos de desarrollo rural. 


\begin{tabular}{|c|c|c|}
\hline Grupos & Sudgrupos & Ejemplos \\
\hline \multirow{4}{*}{ 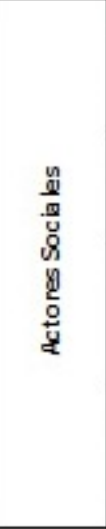 } & $\begin{array}{l}\text { Asociaciones orientadas } \\
\text { a la representación, } \\
\text { defensa y reivindicación } \\
\text { de intereses }\end{array}$ & $\begin{array}{l}\text { Sindicatos de trabajadores y organizaciones profesionales, } \\
\text { Federaciones de cooperativas, Organizaciones de } \\
\text { consumidores, Asociaciones ambientalistas, Asociaciones de } \\
\text { mujeres, de jóvenes, Redes de Desarrollo Rural... }\end{array}$ \\
\hline & $\begin{array}{l}\text { Asociaciones orientadas } \\
\text { a la actividad } \\
\text { económica. }\end{array}$ & $\begin{array}{l}\text { Cooperativas, Sociedades comerciales, Asociacionesu } \\
\text { organizaciones de productores, Consorcios empresariales. }\end{array}$ \\
\hline & $\begin{array}{l}\text { Asociaciones orientadas } \\
\text { a la actividad cultural, } \\
\text { religiosa, recreativa, } \\
\text { ocio,... }\end{array}$ & $\begin{array}{l}\text { Asociaciones civico-culturales, deportivas,..., Asociaciones } \\
\text { religiosas (cofradías, hemmandades), Asociaciones recreativas } \\
\text { (peñas, clubs,...) }\end{array}$ \\
\hline & $\begin{array}{l}\text { Asociaciones orientadas } \\
\text { a la gestión y } \\
\text { promoción del } \\
\text { desarrollo local }\end{array}$ & $\begin{array}{l}\text { Grupos de acción local (iniciativa Leader), Grupos de desarrollo } \\
\text { rural (programa Proder). Redes regionales, nacionales, } \\
\text { internacionales de GAL. }\end{array}$ \\
\hline \multirow{4}{*}{ 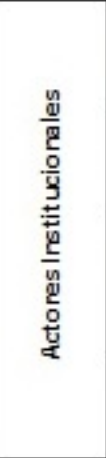 } & $\begin{array}{l}\text { Instituciones públicas } \\
\text { orientadas a la gestión } \\
\text { de las políticas locales }\end{array}$ & $\begin{array}{l}\text { Organismos para la gestión y prestación de servicios } \\
\text { municipales (OM IC,...), Sindicats mixtes entre communes, }\end{array}$ \\
\hline & $\begin{array}{l}\text { Entidades locales de } \\
\text { cooperación } \\
\text { intermunicipal }\end{array}$ & $\begin{array}{l}\text { Mancomunidades, consorcios, mancomunauté de communes, } \\
\text { pays, sindicat mixte, pays d'aglomeration, comarcas, zonas } \\
\text { periurbanas... }\end{array}$ \\
\hline & $\begin{array}{l}\text { Organismos periféricos } \\
\text { de las administraciones } \\
\text { regionalesy nacionales }\end{array}$ & $\begin{array}{l}\text { Centros de salud, centros educativos y de formación, Centros } \\
\text { de servicios sociales, Oficinas comarcales agrarias, Servicios de } \\
\text { medio ambiente (SEPRONA), Parques Naturales y Espacios } \\
\text { Protegidos. }\end{array}$ \\
\hline & Instituciones privadas & $\begin{array}{l}\text { Entidades financieras (bancos, cajas,...), Medios de } \\
\text { comunicación (prensa, TV, radio), Fundaciones, Entidades } \\
\text { religiosas, Entidades educativas y de formación profesional. }\end{array}$ \\
\hline
\end{tabular}

Tipología de Actores en las zonas rurales

La tabla anterior puede ser una muestra de esta gran variabilidad de actores que en la aplicación de un enfoque integral, participativo y territorial son movilizados en los procesos de desarrollo rural actual.

De este modo, la experiencia de las iniciativas LEADER, debido a su largo recorrido con más de 20 años de aplicación, a su enfoque innovador y a la gran cantidad de experiencias locales (16) realizadas se ha convertido en un "laboratorio pan-europeo" (Ray 2000) de soluciones innovadoras para el desarrollo. Las experiencias de los numerosos GAL en la aplicación de tal enfoque fuertemente basado sobre la contribución de los actores locales, aunque no reproducibles, constituyen una mina de informaciones para la investigación, sobre todo comparativa, sobre el desarrollo rural.

La inclusión del método LEADER como eje transversal de actuación del segundo pilar de la PAC (desarrollo rural) supone un paso de especial relevancia para la institucionalización (17) de los procesos de desarrollo rural en Europa. Este cambio supone otorgar carácter orgánico a este método y dotarlo de estabilidad, proyección en el tiempo y reconocimiento (18). Con la creación de este eje se institucionalizan lo que fueron iniciativas de carácter piloto y experimental que han marcado un verdadero "punto de inflexión" (Barrero 2010: 164) en la transformación de los espacios rurales, los Grupos de Acción Local adquieren por ello un mayor reconocimiento y un cambio fundamental en su status como interlocutores directos con la administración, dotados además de un mayor poder de decisión. La relevancia de los GAL en los procesos de desarrollo rural se basará en la capacidad de estas organizaciones para aplicar los principios básicos de proximidad y construcción de redes y vínculos, propios del enfoque LEADER.

"La proximidad permite, en una primera fase, aprovechar los recursos locales y crear un tejido social allí donde parecía imposible. En una segunda fase, la creación de vínculos entre las acciones realizadas, los agentes implicados, e incluso entre los territorios rurales, permite situar a los agentes y al territorio en una escala más global de la economía, a través de la constitución de una masa crítica pertinente" (Bandeira et al. 2004).

El principal potencial de los GAL estriba, por tanto, en su capacidad de movilización y dinamización social de los actores locales, en las funciones de representación de los distintos actores y de 
concertación de sus intereses respectivos y en favorecer las interrelaciones entre los actores a través de la creación de redes. Los GAL, como partenariados horizontales público-privados, supone en sí mismo un espacio de concertación plural entre los actores locales que lo componen, siendo además nodos centrales en redes de coordinación y cooperación entre organizaciones y administraciones públicas del territorio.

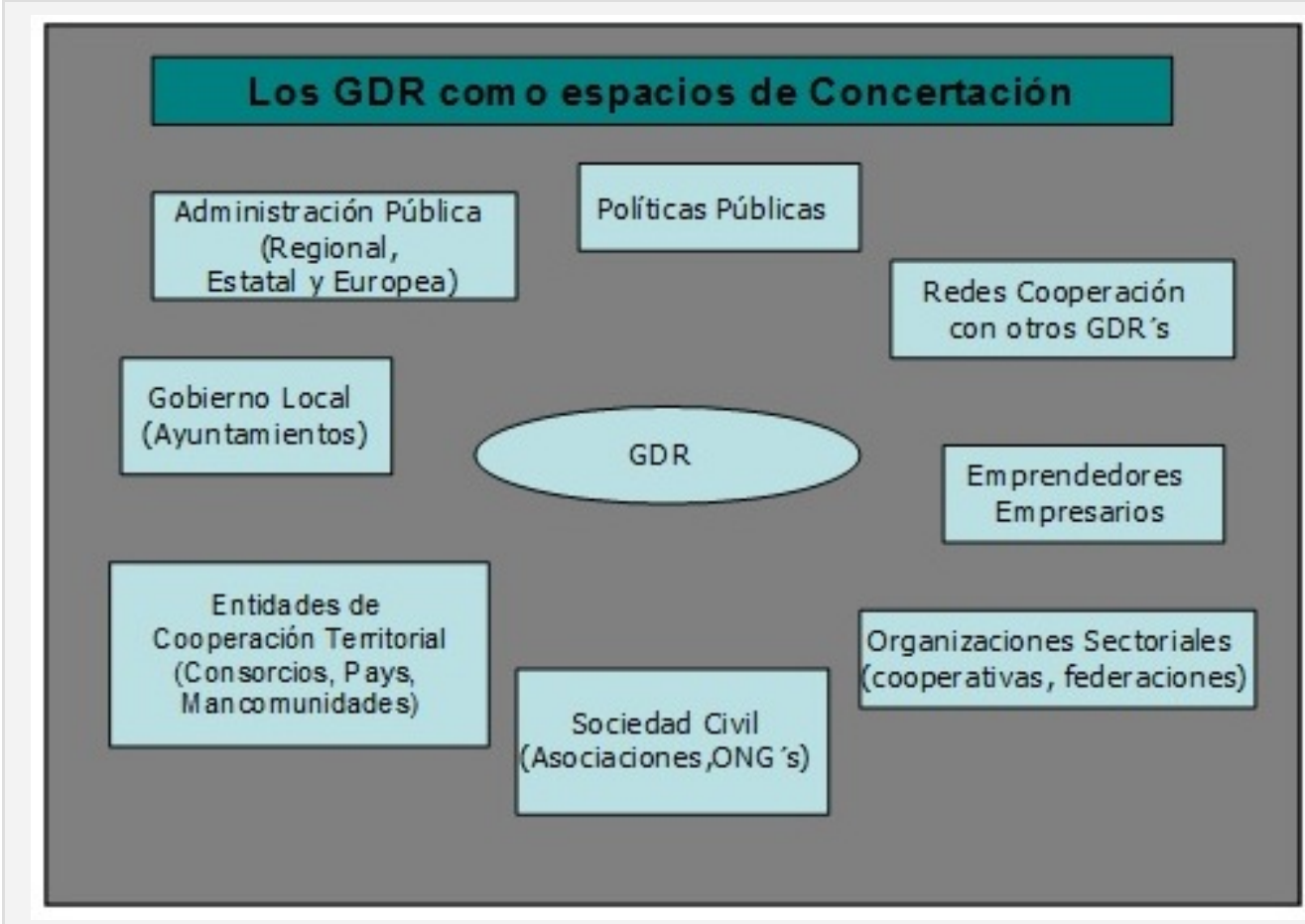

Redes de actores en el Desarrollo Rural

Se configuran redes en las que se dan cita actores intra y extra territoriales siendo ello un reflejo en la dinámica de trabajo en red de esa "tercera vía" (Marsden 1998) en los que los recursos, también los recursos humanos, tanto endógenos, como exógenos deben ser movilizados (Lowe et al. 1997).

Aunque autores especializados confirman el buen papel desarrollado por los GAL en las tres fases de implantación del LEADER señalando que: "Estos grupos constituyen, además, un importante patrimonio social y cultural de los territorios rurales españoles“ (Moyano 2009:18). Apuntan, por otro lado, una posible dificultad en la falta de planificación clara de los espacios de concertación en el nuevo reglamento FEADER y aseguran que la consolidación de los GAL y del enfoque transversal LEADER provocará la existencia de un sistema de gobernanza en el territorio que debería ser fomentado y asegurado por los poderes públicos.

El nuevo status que adquieren los GAL en la actual política de desarrollo rural europea se entiende como un "nuevo reto" (Moyano 2009) en cuanto al fomento de la participación de muy distintos grupos de actores y la consolidación de dinámicas de concertación entre ellos. Lo que no resultará fácil teniendo en cuenta que deben ser modificados los espacios de decisión que estos distintos grupos han desarrollado "tradicionalmente" a través de la aplicación de políticas específicas, como en el caso de los agricultores. Con estos cambios, referidos a la obligación de concertación entre actores de las zonas rurales, se ponen en riesgo los espacios de poder e influencia de estos actores (Esparcia y Noguera 1999) y se enfrentarán las distintas concepciones del desarrollo rural que cada uno de estos actores representa.

La implicación o activación de los actores dependerá de una simbiosis entre factores tan importantes y diversos como la memoria colectiva, la identidad común, la voluntad local de superar los problemas y la apertura al entorno exterior. El objetivo, por tanto, dentro de estos "sistemas institucionales" para el desarrollo rural será definir un proyecto común en función de la imagen que la sociedad local tiene de ella misma y de su futuro, es decir de los modelos y de los valores que la distinguen (Houée 1992). Dicho de otro modo, la convergencia o la síntesis de las decisiones individuales depende de que exista o no un 
argumento aglutinador capaz de concentrar las capacidades del sistema (Ramos y Romero 1995) y de consensuar en red ciertos elementos federadores que refuercen ese proyecto, tales como la propia concepción del Desarrollo o la construcción consensuada de la imagen del Territorio y sus potencialidades.

\subsection{La dimensión territorial del desarrollo rural}

Una de las consecuencias más visibles del nuevo enfoque de aplicación de las políticas de desarrollo rural europeas guiadas por la metodología LEADER ha sido, como ya se ha señalado, la constitución y reconocimiento de nuevas redes de actores diversos implicados en la planificación y la gestión del desarrollo rural. En paralelo la constitución de estas redes de actores emergen en las zonas rurales nuevos ámbitos espaciales de actuación. A través del impulso dado a los GAL y a la metodología participativa y territorial se han generado nuevas demarcaciones espaciales, nuevos territorios que es necesario redefinir, revalorizar y distinguir, dando con ello sentido a un conjunto de actuaciones e iniciativas exclusivas de desarrollo rural.

La emergencia de estos nuevos territorios ha sido un fenómeno generalizado en la UE, si bien comenzó a aplicarse a través de la primera iniciativa LEADER en ciertos espacios rurales seleccionados, dentro de las zonas objetivo 1 y 5b, este modelo se ha ido generalizando y expandiendo hasta la inclusión del enfoque LEADER como eje transversal de la política de desarrollo rural, segundo pilar de la PAC.

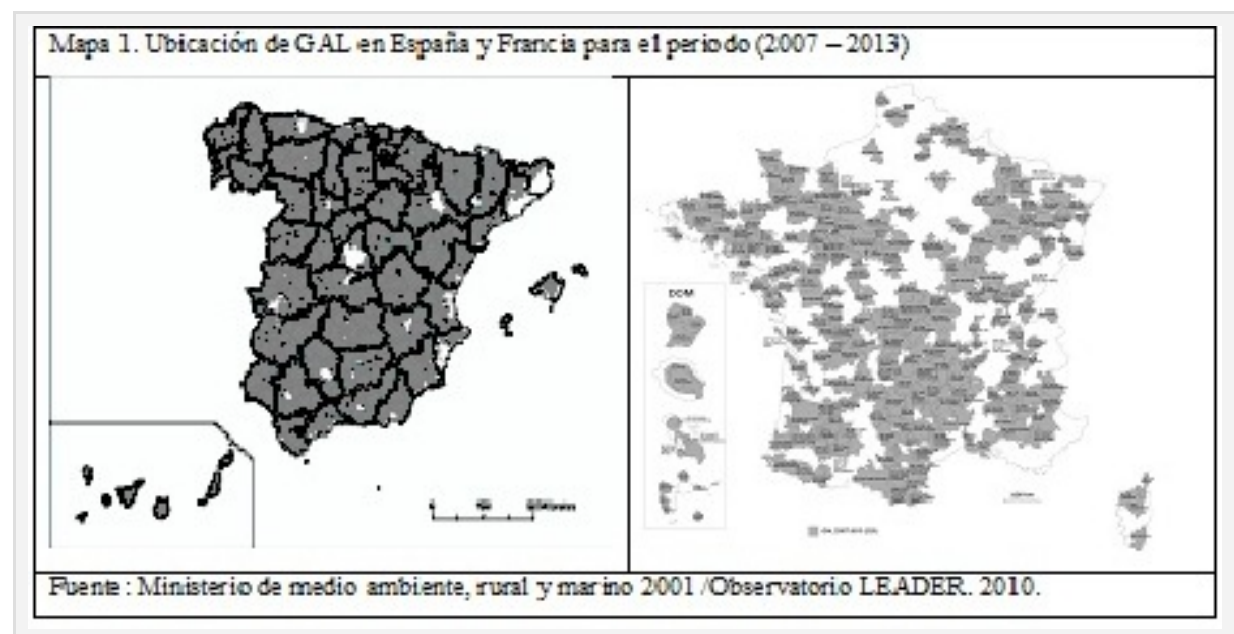

A modo de ejemplo, vemos como en los casos de España y Francia, la repercusión a nivel territorial de los programas de desarrollo rural ha sido enorme. En España estos programas afectan al $90 \%$ de los municipios, un $88 \%$ del territorio nacional donde habita un $26 \%$ de la población. Pero lo más importante es que se consolidan, durante la programación FEADER (2007-2013) 264 GAL que gestionan la realización de estas políticas en nuevas demarcaciones en las que quedan divididas las zonas rurales. Del mismo modo, en Francia, son 223 los GAL aprobados para este mismo periodo que afectan a un $64 \%$ del territorio nacional.

Entender este nuevo mapa es fundamental para explicar la profunda transformación del escenario rural europeo en las tres últimas décadas. Dado que, estas nuevas organizaciones (GAL) y las redes de actores creadas en torno a ellas, han instrumentalizado a nivel local, las grandes líneas del desarrollo generadas desde el ámbito supranacional europeo, haciendo valer para ello la profunda imbricación que estos nuevos modelos de desarrollo tienen en sus respectivos territorios, mediante la experiencia aportada por programas anteriores entre los que destaca la Iniciativa LEADER.

Con la consolidación de los GAL se impulsa un nuevo ámbito de intervención intermedio, el ámbito supramunicipal alcanza cada vez una mayor relevancia en la gestión del desarrollo y la ordenación del territorio (Precedo Ledo 2006, Infante 2010, Gómez Orea 1993, Hervieu 1995). En este sentido, Hervieu enuncia del modo siguiente la necesidad de establecer este nuevo ámbito de intevención:

"El municipio rural multisecular ya no constituye un escalón suficiente de desarrollo y ordenación. Un 
sistema de cooperaciones intermunicipales flexibles debe permitir trabajar conjuntamente a entidades territoriales comparables" (Hervieu 1995: 37).

La intervención en ámbitos supramunicipales o comunitarios responde a la aplicación de una visión o enfoque territorial del desarrollo. Con esta misma orientación distintas políticas realizadas en los Estados Miembros sobre la ordenación territorial, la descentralización y la cooperación intermunicipal han fortalecido la definición de estos nuevos espacios rurales de actuación. Ejemplo de ello pueden ser las distintas políticas nacionales específicas para "zonas de montaña", los contrats de pays franceses, los contratti d'area italianos o las políticas de Dorferneuerung (renovación de pueblos) en Alemania, así como los procesos de comarcalización iniciados en algunas CCAA españolas (Aragón, Cataluña o Galicia). Con estas políticas se crean nuevas entidades de cooperación intermunicipal (mancomunidades, comarcas, pays) en este nivel comunitario que asumen la promoción del desarrollo como función, será por ello esencial analizar las articulaciones entre estas entidades y los GAL y la variabilidad de modelos que estas articulaciones puede conllevar.

La aplicación del enfoque territorial a las políticas de desarrollo conlleva la definición de una política y un conjunto de estrategias en función de las características particulares de cada territorio. De este modo, el territorio pasa de ser un "soporte" en el que aplicar iniciativas de desarrollo a ser un "recurso" esencial, puesto que son sus propias características las que definen las estrategias a seguir (Esparcia y Noguera 1999).

Esta visión territorial del desarrollo implica la realización de un proceso de redefinición territorial en el que intervienen una compleja red de actores sociales e institucionales. Uno de las más importantes transformaciones ligadas al desarrollo rural es la construcción de estos nuevos territorios, en la que se define un proyecto territorial de desarrollo exclusivo para cada territorio, con el que se intenta garantizar la competitividad territorial de cada uno de ellos. Esta competitividad se basa en otorgar al territorio un "valor añadido" mediante sus recursos específicos, para lo cual será esencial la labor de promoción y marketing territorial (Caroli 1999) que aquellos sistemas institucionales (dimensión organizativainstitucional) realicen, creando una imagen corporativa del territorio en la que se articulen sus potencialidades para el desarrollo y elementos federadores con los que la población se sienta identificada.

De este modo se genera un producto, una "imagen del territorio" en la que se conjugan los intereses y expectativas de los distintos actores locales y que debe ser atractivo hacia el exterior para conseguir atraer externalidades positivas que garanticen el proceso de desarrollo.

Hacemos especial énfasis en señalar que este proceso de construcción territorial se realiza a través de nuevas dinámicas sociales en red de los actores locales. En este proceso existe una interacción entre los actores y el territorio (19) puesto que se trata de un proceso de vital importancia para la realización de estos proyectos territoriales y, a su vez, consolidan estas redes de organizaciones sociales implicadas en la planificación del desarrollo.

\subsection{La Dimensión identitaria del desarrollo rural}

Son muchas las voces que, provenientes de distintas disciplinas, han venido insistiendo en la importancia que los aspectos culturales tienen en los procesos de desarrollo. Como afirma Boisier (2003) la continua evolución del concepto de desarrollo han hecho que se incorporen en su definición elementos de ámbitos distintos al económico, esta tendencia denominada por diversos autores como el "giro cultural" del desarrollo (Tomassini 2000). Desde nuestra perspectiva de análisis el papel de la identidad cultural en los actuales procesos de desarrollo de las zonas rurales europeas es central, puesto que la identidad cultural es reconocida como un elemento clave de estos procesos. El valor y los roles que la identidad colectiva juega en estos procesos es múltiple y está directamente relacionada con las dimensiones organizativa y territorial de estos procesos.

Por un lado, la identidad colectiva aporta ciertos elementos culturales que van a dar sentido al territorio y que lo dotan de especificidad. En procesos donde la imagen territorial basa su valor añadido en la 
especificidad del territorio, la referencia a los elementos culturales es ineludible puesto que son estos los que aportan tal especificidad. Esta función que hemos denominado de especificad territorial, hace que la imagen del territorio tanto en el interior (para la propia población), como en el exterior (como "producto" en el mercado) sea asociada a ciertos elementos culturales que la hacen única. A través de esta función se aplica al territorio una estrategia análoga a aquellas de la economía cultural (Ray 1998), en las que determinados productos, saberes o formas de producción o elaboración funcionan como marcadores de identidad y aportan un valor añadido a estos productos en el mercado.

Por otro lado, la existencia de una identidad colectiva fortalece la idea de comunidad, el sentimiento de pertenencia y la cohesión social. Por ello la identidad colectiva, articulada a un proyecto territorial de desarrollo puede reforzar los nuevos modelos de organización encabezados por los GAL. Una identidad colectiva fuerte fomentará la percepción del territorio comarcal como algo propio, del tal modo que fortalece la implicación de los actores en los procesos de toma de decisiones reforzando, legitimando y dando sostenibilidad social (Güell 2001) al proceso en la medida en que la población se siente representada y apoya estas iniciativas.

Teniendo en cuenta las funciones que la identidad colectiva tiene para los procesos de desarrollo rural, nuestro principal interés de investigación será analizar las distintas acciones y estrategias elaboradas para fortalecer la identidad colectiva, los resultados obtenidos y los límites y obstáculos que pueden presentar este tipo de estrategias.

El desarrollo rural en red. El abordaje de las distintas dimensiones de transformación de los procesos de desarrollo desde el análisis de redes sociales.

Las distintas dimensiones de los procesos de desarrollo presentadas en el apartado anterior evidencian lo complejo y plural que son estos procesos y lo alejados que éstos se encuentran de una acepción centrada en el crecimiento económico. Efectivamente el desarrollo es mucho más que crecimiento económico, los propios economistas ya hace mucho tiempo que han reconocido este aspecto y han elaborado métodos de análisis del desarrollo centrado en factores sociales y culturales como causa de este proceso (Amin 1998, Amin y Thrift 1993).

"el verdadero desarrollo es principalmente un proceso de activación y canalización de fuerzas sociales, de avance en la capacidad asociativa, de ejercicio de la iniciativa y de la inventiva. Por lo tanto, se trata de un proceso social y cultural, y sólo secundariamente económico. Se produce el desarrollo cuando en la sociedad se manifiesta una energía, capaz de canalizar, de forma convergente, fuerzas que estaban latentes o dispersas" (Furtado 1982: 149).

A una conceptualización del desarrollo como proceso complejo han seguido distintas propuestas teóricas que son capaces de abordar esta complejidad. El análisis de redes sociales constituye en esta línea un nuevo paradigma para el análisis del desarrollo (Murdoch 2000). Un análisis de redes en el ámbito del desarrollo puede interpretar, por un lado los campos de relaciones articulados en torno al concepto de desarrollo en sus múltiples dinámicas de afirmación, traducción y contestación; y, por otro lado, los procesos a través de los cuales los elementos materiales y simbólicos que constituyen la realidad social son transformados, reproducidos, instituidos o contestados (Job Schmitt 2011).

La "perspectiva orientada en los actores" (Long 1992, Ploeg 1990, Ploeg y Marsden 2008) plantea un enfoque de análisis de redes aplicado a los procesos de desarrollo e intenta analizar la influencia de los distintos agentes en la implementación de políticas y proyectos de desarrollo. Lo esencial en este enfoque es que partiendo del supuesto de que los cambios estructurales son resultado de la acción del Estado, el Mercado o las políticas de desarrollo (las denominadas "fuerzas externas"), las formas de intervención consecuencia de estos cambios sólo afectaran a las oportunidades sociales y a la conducta de los individuos en la medida en que se introduzcan en sus modos de vida, afectando de forma directa o indirecta a sus experiencias cotidianas (Long 2007).

Una crítica fuerte a la idea de la "intervención planeada" y un énfasis en la experiencia social construida en la vida cotidiana son componentes de este abordaje, que busca aprehender, desde una perspectiva 
constructivista "las maneras intrincadas y variadas en que viejas y nuevas formas de producción, consumo, sustento e identidad se entrelazan y generan modelos heterogéneos de cambio económico y cultural" (Long 2007: 39).

Desde este enfoque, los actores canalizan a través de cadenas distintos elementos (información, demandas), estas cadenas constituyen un conjunto de puntos nodales de interpretación sobre estos elementos y de interacción entre los actores. De este modo vemos como en la red se movilizan relaciones, recursos y también significados e interpretaciones que propician la interrelación entre "mundos diferentes", entre distintas formas de conocimiento, trascendiendo dominios institucionales específicos y conectando una gran diversidad de arenas. La confluencia de estos múltiples elementos se realiza en la red y es en la red en la que a través de procesos de lucha, negociación y adaptación se construye el propio desarrollo y ciertas representaciones sociales compartidas por los miembros de la red que guiaran el proceso.

Una interesante propuesta teórica reciente ha intentado integrar los distintos enfoques del análisis de redes aplicado al ámbito del desarrollo en zonas rurales, hablamos del programa de investigación ETUDE (20). En este interesante trabajo las redes han sido incorporadas como un instrumento de análisis, que posibilite el estudio de las transformaciones del espacio rural contemporáneo; y como parte de un cuerpo teórico y metodológico que pueda dirigirse a la implementación de políticas capaces de orientar estrategias de desarrollo rural sostenible.

Las redes que conforman las sociedades y las economías rurales surgen de la combinación de diferentes elementos: actores, actividades, lugares, recursos... que en su articulación darán origen a distintas trayectorias de desarrollo evidenciando una gran diversidad de configuraciones del espacio rural. Desde esta perspectiva, las redes constituyen un modelo analítico tanto como un recurso heurístico, que permite "fotografiar" las distintas configuraciones asumidas por aquellas dimensiones analíticas (endogeneidad...) en procesos regionales de desarrollo rural. Pero la propuesta de investigación que proponen no constituirá una mera descripción de la multiplicidad y heterogeneidad de trayectorias de desarrollo, será preciso también comprender el modo en que los factores externos (contra-urbanización, globalización...) influyen en el desarrollo de las regiones rurales (Horlings y Marsden 2010).

Adoptando este enfoque de redes para el análisis de los procesos de desarrollo rural nuestra perspectiva de investigación se centra en analizar y comprender las aportaciones realizadas por el conjunto de actores implicados en estas redes en cada uno de los casos analizados, pudiendo con ello definir su modelo específico en dos aspectos esenciales. El primero de los aspectos que nos interesa analizar de estas redes es su capacidad para la movilización de actores y recursos y la constitución de relaciones entre ellos, para lo cual utilizaremos el concepto de capital social (Woolcock 1998). El segundo de los aspectos en nuestro análisis lo constituyen los procesos de construcción generados desde estas redes en los que se definen normas, reglas y representaciones colectivas que orientan el proceso en cada caso específico, será para ello necesario atender al concepto de capital institucional (Garrabé 2007).

La definición que aporta Woolcock sobre el capital social lo define como "las redes que permiten la acción colectiva" en la medida en que "fomentan la creación de vínculos entre los actores". El modelo de análisis de Woolcock se basa en la existencia de cuatro tipos de capital social que pueden aportar beneficios a la realización de procesos de desarrollo y que se encuentran en niveles distintos, el nivel micro que se refiere a las relaciones entre los individuos o grupos dentro de redes, y el nivel macro, referido a las instituciones (21).

En el nivel micro se distinguen dos tipos de capital social. La Integración Intracomunitaria (integration): se basa en los vínculos o relaciones estrechas que existen dentro de la comunidad. Este tipo puede referirse a las relaciones entre la población de una comunidad, a un determinado sector dentro de ella. Las Relaciones Extracomunitarias (linkage): se basa en las relaciones que se dan fuera de la comunidad, entre los individuos o grupos locales y otos fuera del territorio que se analiza.

En el nivel macro se presentan otros dos tipos de capital social: La Sinergia Institucional, comprende las 
relaciones entre las instituciones (organizaciones) y entre ellas y los grupos o colectivos locales en el territorio analizado. La Eficiencia Organizacional se basa en criterios de capacidad y credibilidad institucional; es decir, en el grado de eficiencia que son capaces de realizar las instituciones que tienen que jugar un papel en el desarrollo, y en cómo son valoradas por la sociedad, su capacidad de generar confianza, su reputación.

A través de este análisis del capital social podemos profundizar en la dimensión organizativa del desarrollo que hemos presentado, así como en la dimensión identitaria del mismo, en la medida en que la eficiencia organizacional remite al grado de confianza de la población y a su identificación con el proyecto territorial.

Según Garrabé, "el capital institucional representa el conjunto de instituciones formales e informales que constituyen la estructura que organiza las relaciones entre individuos o entre las organizaciones, en el seno de procesos de producción económica y social" (Garrabé 2007: 5). Para nuestra investigación el capital institucional proporciona ciertos elementos de interés, ya que desde un enfoque dinámico y procesual permite una reflexión sobre la construcción y la movilización de reglas que orientan las relaciones entre los actores implicados y la existencia de ciertos valores comunes y representaciones colectivas que orientan la movilización de recursos y la definición de estrategias específicas para el desarrollo rural en un contexto determinado.

Tal y como plantea Ostrom $(1992,1998)$, existen instituciones exteriores a la acción colectiva y otras instituciones que son propias a la acción colectiva. Las instituciones exteriores forman parte del marco socio - económico y político que se impone a esta acción colectiva, estas instituciones generalmente existen previamente a la realización de la acción, aunque como indica la autora pueden llegar a ser modificadas por esta acción colectiva. En lo que respecta a las instituciones propias a la acción colectiva, estas son las normas, que son valores internos al grupo, y las reglas, que constituyen representaciones colectivas. Tanto unas como las otras se van construyendo mediante la acción colectiva y se ponen en funcionamiento progresivamente permitiendo a los distintos actores trabajar juntos.

El análisis del capital institucional aplicado a nuestra investigación se centra en la construcción de estas instituciones propias a la acción colectiva, entre las que diferenciaremos las formales e informales, tal y como propone Garrabé. Una de las instituciones propias formales sería, por ejemplo, el Plan de Desarrollo Territorial como documento de diseño y planificación del desarrollo realizado mediante la acción colectiva en red. Mientras que existen otras instituciones propias informales que deben ser analizadas de forma complementaria, como son el Territorio, la "Imagen Territorial" o la propia idea de comunidad. Ambas configuran reglas, representaciones colectivas emanadas de esa acción colectiva y el interés será analizar su proceso de definición.

Mediante el análisis del capital institucional podemos abordar tanto la dimensión organizativa e institucional, como las dimensiones territorial e identitaria de los procesos de desarrollo. Vemos como en los procesos de desarrollo rural europeos motivan la acción colectiva, a través de la cual se forman redes de relaciones entre actores y organizaciones locales (capital social) para planificar estas estrategias. En la dinámica de actuación estas redes se movilizan recursos (entre otros la identidad) sobre los que definir una estrategia de desarrollo en base a una "Imagen del Territorio" (redefinición territorial) que es construida por esta red conformando una representación colectiva (capital institucional) que orientará las acciones concretas de desarrollo implementadas en ese contexto.

La principal aportación de esta propuesta teorico metodológica a la hora de abordar el análisis de procesos de desarrollo rural es su capacidad de transferencia y aplicabilidad pese a las grandes diferencias existentes entre la multitud de experiencas realizadas en el marco de la Unión Europea. A través de un modelo de análisis como el propuesto en estas páginas se abordan las principales transformaciones sociales impulsadas por los procesos de desarrollo rural. De este modelo de análisis general abordamos las dinámicas de acción colectiva, con la activación y movilización de redes de actores locales para la planificación del desarrollo en sus territorios, con tal objetivo estos territorios son reconstruidos o redefinidos, por estas mismas redes, en productos o imágenes para el mercado 
globalizado y cuyo valor diferencial nace de la reinterpretación, reinvención o recuperación de elementos culturales propios. Un enfoque como este puede abordar una enorme variabilidad de casos divergentes aportando una gran riqueza a la investigación sobre desarrollo en la actualidad.

\section{NOTAS}

1. La investigación de la que es fruto esta comunicación es uno de los resultados del desarrollo de los programas I+D titulados "Territorio, calidad e innovación: El diseño de la nueva ruralidad europea". I+D (SEJ2007-63537/SOCl) y "La producción de calidad: nuevas estrategias rurales para nuevos consumidores" Ministerio de Educación y Ciencia.

2. Esta corriente es desarrollada por un grupo de sociólogos europeos, como principales impulsores de esta corriente teórica de la sociología rural destacan autores como Terry Marsden (Marsden et al. 1990 y Marsden 1992), Philip Lowe (Lowe et al. 1993).

3. Este proceso de Reestructuración de las zonas rurales es presentado del siguiente modo por esta corriente teórica: "las áreas rurales, sus residentes y organizaciones se enfrentan en la actualidad a un rápido cambio social, económico y político. El balance entre producción, ocio, movilidad y desarrollo se reajusta en la medida que las actividades económicas se relocalizan. Igualmente los valores relativos a la vida y la participación en lo rural son objeto de cambio. Fuerzas políticas locales, nacionales e internacionales tienen una influencia directa sobre las áreas rurales" (Marsden et al. 1990).

4. La publicación de la obra "Constructing the Countryside" (Marsden et al. 1993) supone el origen de esta nueva corriente teórica que desde la sociología rural aborda las transformaciones de las zonas rurales en Europa. Algunos autores como los ya citados Marsden y Lowe, serán referentes también de esta nueva corriente constructivista aplicada a la sociología rural, sin embargo, otros autores como John Urry o Heith Halfacree serán los responsables de dotar a esta corriente de un cuerpo teórico distintivo y renovador con respecto a la corriente de la reestructuración rural, tal como hemos intentado explicar en el texto.

5. En la configuración de los espacios rurales como construcciones sociales, esta corriente muestra una influencia clara de las propuestas teóricas que Berger y Luckman recogieron en su obra "La construcción social de la realidad" (1986).

6. En este sentido Oliva y Camarero exponen que lo rural "como elemento imaginario asociado a lo natural, la salud, la identidad, la pertenencia... 'lo rural' pasa a formar parte de nuestras estrategias identitarias, de consumo, residenciales, turísticas, etc." (Oliva y Camarero 2002: 26).

7. El reconocimiento de la multifuncionalidad de la agricultura supone el reconocimiento a todos los niveles de su contribución además de al ámbito económico, a los ámbitos culturales (paisaje cultural, explotación tradicional), sociales (fuente de la estructuración y organización social tradicional) y medioambientales (biodiversidad y sostenibilidad ambiental).

8. Con la noción de competitividad territorial se hace énfasis en la necesaria especificidad que deben tener estos activos para poder otorgar un valor añadido a los productos (o los territorios) en el mercado global. Del mismo modo hace patente la complementariedad entre estos activos específicos con ciertas externalidades positivas en el refuerzo de estos procesos económicos (innovación y nuevas tecnologías, inversión, calidad y procesos de certificación) en un proceso mixto y único en el que se articulan las virtudes asociadas tanto al desarrollo endógeno, como al exógeno.

9. El contenido de este artículo se basa en la realización de un trabajo de investigación encaminada a la 
realización de una Tesis Doctoral, en el que se han analizado, mediante un análisis etnográfico, dos casos en dos países de la Unión Europea: La comarca de Guadalteba en la Comunidad Autónoma de Andalucía (España) y el Pays Gorges, Causses, Cévennes, en la región de Languedoc-Roussillon (Francia). Por ello es imprescindible para este tipo de investigación el análisis del contexto y la selección de ciertas dimensiones y elementos comparables que sostengan la realización de una comparación de los procesos de desarrollo realizados en cada uno de ellos.

10. Esta publicación es uno de los frutos de un proyecto conjunto entre diversas universidades europeas titulado "The socio-economics impact of rural develpoment policies: realitis and potentials (CT-4288) desarrollado en el marco del cuarto programa marco de investigación FAIR.

11. LEADER (Liaisons Entre Actions de Développement de l'Economie Rurale) constituye el primer instrumento directo de desarrollo rural promovido en el marco de la UE, en su primera fase, nace como una Iniciativa comunitaria (IC) creada por la Comisión Europea dentro del marco de la Política Regional Comunitaria. En esta fase su objetivo fue generar experiencias piloto y efectos demostrativos de desarrollo rural. Tras la realización de tres fases sucesivas entre los años 1991 y 2006 esta iniciativa consolidó lo que ha venido denominándose como método, enfoque o filosofía LEADER. Durante sus 15 años de historia, esta IC ha demostrado la valía de su método, hasta el punto de llegar a insertarse orgánicamente en la programación ordinaria de desarrollo rural de la UE, constituyendo una parte del segundo pilar de la Política Agraria Común (PAC). De este modo en el Reglamento sobre desarrollo rural realizado para el periodo 2007-2013, se introduce el nuevo eje IV que tiene como objetivo "Construir la capacidad local de ocupación y diversificación" y se basado en el enfoque LEADER y que actúa como eje transversal en este reglamento. Partiendo de ser una IC el LEADER ha pasado a formar parte integrante en la actual PAC.

12. En este caso el sentido de "institucional" se refiere a las entidades de administración pública y no a la definición analítica aportada en este artículo. Se refiere al entorno conformado por las corporaciones locales, los ayuntamientos, las entidades de cooperación interterritorial y por entidades administrativas descentralizadas pertenecientes al gobierno regional o nacional que trabajan temáticas muy directamente ligadas al desarrollo rural (empleo, educación, agricultura...).

13. En el apartado 4 del Artículo 62 (Grupos de Acción Local) del Reglamento (CE) No 1698/2005 del Consejo (Reglamento FEADER) se especifican las funciones que deben asumir los GAL que serán de dos tipos. Por un lado, realizarán las funciones que determine el plan, programa o actuación que ejecuten, por otro lado ejercerán ciertas funciones como entidades que participan en la gestión y ejecución del Eje 4 de la Política de Desarrollo Rural, refereridas al asesoramiento, valoración y concesión de las solicitudes de subvención.

14. En referencia a este tipo de "logros" conseguidos con la aplicación de los programas LEADER, se alude en numerosas ocasiones a un aumento significativo del capital social en las zonas rurales, aunque en nuestra opinión no se haya realizado un análisis pertinente en estas evaluaciones que sostenga esta afirmación.

15. Los actores institucionales son aquellos que tienen alguna relación con la administración pública. En muchas tipologías de actores utilizadas para la investigación de procesos de desarrollo rural se utiliza esta primera distinción tipológica entre actores sociales, como aquellas asociaciones y organizaciones colectivas representativas de la población local y actores institucionales (Garrido y Moyano 2003).

16. En este sentido el gran impacto de esta iniciativa comunitaria se demuestra al comprobar que la Iniciativa Comunitaria LEADER I se experimentó en 217 territorios dentro de las áreas objetivo 1 y 5 b de la UE, por su parte LEADER II se puso en funcionamiento en 906 territorios pertenecientes a las mismas áreas, y finalmente LEADER+ fue aplicado en 893 territorios situados en cualquier zona europea afectando a una superficie total de $1.500 .000 \mathrm{~km} 2$ y a una población de 53.600 .000 personas. En la mayoría de los países de la UE que fueron beneficiarios de esta iniciativa un porcentaje de entre el 40 y $50 \%$ de su superficie total estaría afectada por esta iniciativa. 
17. Todo proceso de institucionalización viene a referirse a un proceso de transformación en el que una práctica, un grupo, una red... pasan desde una situación informal e inorgánica hacia otra situación más organizada, con una práctica estable que será interpretada como la labor propia de una entidad dotada de personalidad jurídica que tenga continuidad y proyección en el tiempo.

18. El considerando (50) del Reglamento (CE) No 1698/2005 del Consejo (Reglamento FEADER) expone del siguiente modo la inclusión del enfoque LEADER en el marco político comunitario: "Transcurridos tres períodos de programación, la iniciativa Leader ha alcanzado un grado de madurez que permite a las zonas rurales aplicar el enfoque propio de dicha iniciativa en el contexto más amplio de la programación general del desarrollo rural. Así pues, es preciso extender los principios básicos del enfoque Leader a los programas que integren un eje específico y definir los grupos de acción local y las medidas que vayan a ser objeto de ayudas, incluidas la creación de partenariados, la aplicación de estrategias locales, la cooperación, la creación de redes y la adquisición de capacidades".

19. "La interacción entre agentes y lugares (entre actores y territorio) se construye mutuamente en un proceso complejo en el que se entrelazan diversas concepciones del territorio: una concepción de tipo administrativo (el territorio como "espacios de las competencias") una concepción unida a la pertenencia natural dada a los lugares (el territorio como patrimonio o herencia del pasado) y finalmente, una concepción "constructivista" del territorio como "construcción social" que crea identidad local en función y en relación a la acción colectiva de los agentes (el territorio-proyecto)" (Bourdin 1994, citado en Dematteis y Governa 2005).

20. Programa ETUDE. Acrónimo de "Enlarging the understanding of rural development in Europe", cuya traducción en castellano sería "Ampliando la comprensión sobre el desarrollo rural en Europa". En este programa participaron investigadores de 6 países europeos, y fueron reunidos y analizados un amplio conjunto de experiencias de desarrollo rural (63casos) ambientadas en distintas regiones del continente. Los resultados generados por esta iniciativa de investigación interdisciplinar fueron reunidos en dos publicaciones: Unfolding webs: the dynamics of regional rural development, libro organizado por Ploeg e Marsden (2008), y Networking the rural: the future of green regions in Europe una recopilación de artículos organizada por Milone y Ventura (2010).

21. Las "instituciones" a las que se refiere el autor son las organizaciones o entidades que dentro de la red pueden tener una mayor relación con la administración pública o tienen la capacidad de toma de decisiones sobre los procesos. Por ello las entenderemos de forma general como "organizaciones" para no confundirlas con las "instituciones" en el sentido propuesto por North como "reglas del juego".

\section{BIBLIOGRAFÍA}

Aguilar, E. (y otros)

2009 "Entre la tradición y la innovación: políticas de empleo femenino y desarrollo rural", Sociología del Trabajo, nº 65: 111-136.

Amin, A. (y N. Thrift)

1993 "Globalization, Institucional Thikness and Local Prospects", Reveu d'Economie et Urbaine, n 3 : 416-417.

1998 "Una perspectiva institucionalista del desarrollo regional", Ekonomiaz, n $41.2^{\circ}$ Cuatrimestre 1998: 68-89.

Bandeira, P. (I.Atance y J.M. Sumpsi)

2004 "Las políticas de desarrollo rural en América Latina: requerimiento de un nuevo enfoque" en Cuadernos de Desarrollo Rural, n 51: 25-48. 
Barrero Linares, D.

2010 "Los Grupos de Desarrollo Rural y la revalorización de la cultura rural como recurso para el desarrollo" Ph Cuadernos, n² 26: 163-173.

Bradshaw, T. (y E. J. Blakely)

1979 Rural communities in Advanded Industrial Society. New York: Praeger Publishers.

Boisier, S.

2003. “¿Y si el desarrollo fuera una emergencia sistémica?”, Reforma y Democracia, n 27: 1-24.

Bueno, C. (y E. Aguilar)

2003 "Introducción. La globalización de las expresiones locales", en Bueno, C. y Aguilar, E. (Coord) Las expresiones locales de la globalización. México y España: 5-46. México: Ed. CIESAS, Universidad Iberoamericana y Ed. Porrúa.

Camarero, L. (y M. González)

2005 "Los procesos recientes de transformación de las áreas rurales españolas: una lectura desde la reestructuración ampliada", Sociologia - Revista da Faculdade de Letras da Universidade de Porto, $\mathrm{n}^{\circ}$ 15: 95-123.

Caroli, M. G.

2000 /l marketing territoriale. Milán, Franco Angeli.

Consejo de Europa.

2005 "Reglamento (C.E.) 1698/05, relativo a la ayuda al desarrollo rural a través del Fondo Europeo Agrícola de Desarrollo Rural (FEADER)”, D. O. L 277 de 21-10- 2005, Bruselas: 1-40.

Dematteis, G. (y F. Governa)

2005 "Territorio y territorialidad en el desarrollo local. La contribución del modelo Slot" Boletín de la Asociación de Geógrafos Españoles A.G.E., n 39: 31 - 58.

Elder, J.W.

1976 "Comparative Cross-National Methodology", Annual Review of Sociology, n 2: 209-230.

Esparcia, J. (y J. Noguera)

1999 "Reflexiones en torno al territorio y al desarrollo rural", en E. Ramos(coord.) El desarrollo rural en la Agenda 2000: 9 -44. Madrid, MAPA, Serie Estudios número 142.

Farrell, G.

2003 "El valor añadido de LEADER". LEADER Magazines, n²3: 8-21.

Furtado, C.

1982 A nova dependencia. São Paulo, Paz e Terra.

Garrabé, M.

2007 "Production de capital institutionnel par les OESS", FormDer - Formation Multipôle et Pluridisciplinaire en Développement Rural, CIHEAM.

Gómez Orea, D.

1993 “Articulación y equilibrio territorial”, El desarrollo rural andaluz a las puertas del siglo XXI: 163-178. Sevilla, Junta de Andalucía.

González, M.

2001. Sociología y Ruralidades. La construcción social del desarrollo rural en el valle de Liébana. Madrid: MAPA. 
González, M. T. ( y L. A. Camarero)

1999 "Reflexiones sobre el desarrollo rural: las tramoyas de la postmodernidad", Política y sociedad, $\mathrm{n}^{\circ}$ 31: 55-68.

Güell, P. E.

2001 “Subjetividad Social: desafío para el nuevo siglo", Polis (en línea), Vol 1.

Halfacree, K. H.

1993 "Locality and social representation: Space, discourse and alternative definitions of the rural", Journal of Rural Studies, no 9 (1): 23-37.

1997 "Postmodern perspective on counterurbanisation", en O. Cloke, O. y J. Little (eds.), Contested countryside cultures. Otherness, marginalisation and rurality. Londres, Routledge.

Hervieu, B.

1995 "El espacio rural europeo entre la ruptura y el desarrollo" en E. Ramos y J. Cruz, Hacia un nuevo sistema rural: 27-48. Madrid, MAPA.

Horlings, I. (y T. Marsden)

2010 "The new rural paradigm and redefining the rural web" en P. Milone y F. Ventura, Networking the rural: the future of green regions in Europe. Assen-The Netherlands, Van Gorcum: 213-244.

Houée, $P$.

1992 La décentralisation. Territoires ruraux en développement. París, Syros-Alternatives.

Infante, $\mathrm{J}$.

2010 "Aproximación al modelo de comarcalización de Aragón. Una reflexión crítica", Boletín de la Asociación de Geógrafos Españoles, nº 52: 59-80.

Job Schmitt, C.

2011 "Redes, atores e desenvolvimento rural: perspectivas na construçao de uma abordagem relacional", Sociológicas, $\mathrm{n}^{0}$ 27: 82-112.

Lash, S. (y J. Urry)

1998 Economías de signos y espacio: sobre el capitalismo de la postorganización. Buenos Aires, Amorrortu.

Lijphart, A.

1971 "Comparative Politics and the Comparative Method", A. P. S. R., nº 65: 682-693.

Long, $\mathrm{N}$.

1992 Battlefields of Knowledge: the interlocking of thory and practice in social research and development. London and New York, Routledge.

Long, $\mathrm{N}$.

2007 Sociología del desarrollo: una perspectiva centrada en el actor. San Luís de Potosí / México, El Colegio de San Luís / CIESAS.

Lowe, P. (y otros)

1993 "Regulating the new rural spaces: the uneven development of land", Journal or Rural Studies, $\mathrm{n}^{\circ}$ 12: $101-111$.

Lowe, P. (N. Ward y J. Murdoch)

1997 'Redes en el desarrollo rural: más allá de los modelos exógenos y endógenos', Agricultura y sociedad, $n^{\circ}$ 82: 13-43.

Lozano, C. (y E. Aguilar)

2012 "Territorialising organic production: collective actions and public policies in Andalusia". en Sviluppo 
Marsden, T.

1992 "Exploring a Rural Sociology for the Fordist Transition. Incorporating Social Relations into Economic Reestructuring", Sociología Ruralis, nº 32 (2/3): 209-230.

Marsden, T. (y otros)

1993 Constructing the Countryside. London: UCL Press.

Marsden, T.

1998 "New rural territories: regulating the differenciated rural spaces", en Journal of Rural Studies, 14 (1): 107-119.

Marsden, T. (P. Lowe y S. Whatmore)

1990 "Introduction: Questions of Rurality", en T. Marsden, P. Lowe y S. Whatmore (eds.), Rural Restructuring. Global processes and their responses: 1 -20. London, David Fulton.

Milone, P. (y F. Ventura)

2010 Networking the rural: the future of green regions in Europe. Assen-The Netherlands, Van Gorcum.

Mormont, M.

1990 "Who Is Rural? or, How To Be Rural: Towards a Sociology of The Rural", en T. Marsden, P. Lowe y S. Whatmore (eds.), Rural restructuring: global processes and their responses: 21- 44. London, David Fulton.

Moscoso, D.

2007 "El fin de lo rural. Sobre el ocaso de lo rural y el surgimiento de lo posrural", IX Congreso de Sociología. Barcelona, 13-15 septiembre.

Moyano, E.

2009 "Del desarrollo rural al desarrollo territorial", Documento Foro IESA Diciembre 2009.

http://www.iesa.csic.es/publicaciones/detallarpublicacion/id/226

Murdoch, J. (y A. C. Pratt)

1993 "Rural studies: Modernism, postmodernism and the 'post-rural'", en Journal of Rural Studies, $\mathrm{n}^{\circ} 9$ (4): 411-427.

Murdoch, J.

2000 "Networks - a new paradigm for rural development?" en Journal of Rural Studies, n 16: 407-419.

North, D.C.

1984 Estructura y cambio en la historia económica. Madrid, Alianza Universidad.

1990 Institutions, Institutional Change, and Economic Performance. Cambridge, Cambridge University Press.

Oliva, J. (y L. A. Camarero)

2002. Paisajes sociales y metáforas del lugar. Una exploración de la ruralidad itinerante en Navarra. Pamplona, Universidad Pública de Navarra, D.L.

Ostrom, E.

1992 Crafting Institutions for self-governing irrigation systems. ICS Pres, Institute for Contemporary Studies, San Francisco.

1998 "A behavioral approach to the rational-choice theory of collective action", American Political Science Review, no 92 (1): 1-22.

Pérez Iruela, M. (y otros) 
2000 La nueva concepción del desarrollo rural: Estudio de Casos. Córdoba. CSIC- IESA.

Ploeg, J. D. (y otros)

2000 "Rural Development: From Practices and Polices towards Theory", Sociologia Ruralis, n 40 (4): 391-407.

Ploeg, J. D. (y T. Marsden)

2008 Unfolding webs: the dynamics of regional rural development. Assen-The Netherlands: Van Gorcum.

Precedo Ledo, A.

2006 "Cooperación intermunicipal e identidad territorial en espacios rurales: el futuro de la comarca", Revista de Economía Pública Urbana, nº 6: 113 -149.

Ragin, C. C.

1987 The Comparative Method: Moving beyond Qualitative and Quantative Strategies. Berkeley, Univ. of California Press.

Ramos, E. (y J. Romero)

1995 "Para una concepción sistémica del desarrollo rural", en E. Ramos y J. Cruz (coords), Hacia un nuevo sistema rural. Madrid, MAPA: 29-90.

Ray, C.

2000 "The EU Leader Programme: Rural Development Laboratory", Sociologia Ruralis, $n^{\circ} 40$ (2): $163-$ 171.

Sartori, G.

1970 "Concept Misformation in Comparative Politics", A. P. S. R., n 4: 1033-1053.

Tomassini, L.

2000 "El giro cultural de nuestro tiempo", en B. Kliksberg y L. Tomassini (comp.), Capital social y cultura: claves estratégicas para el desarrollo: 59-100. Buenos Aires, F.C.E./BID.

Woolcock, M.

1998 "Social Capital and economic development: Towards a theoretical synthesis and policy framework", Theory and Society, 27 (2): 151-208. 